\title{
GASTROINTESTINAL STROMAL TUMORS
}

\author{
Bozhkov V., P. Chernopolsky, P. Arnaudov \\ Second Department of surgery, UMHAT "St. Marina" - Varna, Medical university - Varna
}

Reviewed by: Assoc. Prof. V. Ignatov

\begin{abstract}
ABSTARCT
Gastrointestinal stromal tumors are rare neoplasm thought to arise from mesenchymal cell of the gastrointestinal tract. GIST represents $1 \%$ of all primary tumors of gastrointestinal tract; it means they are detected rarely.
\end{abstract}

Key words: gastrointestinal stromal tumors, KIT protein, stem cell factor

Gastrointestinal stromal tumor (GIST) is a recently recognised tumor entity. In the past, these tumors were classified as leiomyomas, leiomyosarcomas and leiomyoblastomas, but it is now evident that GIST is a separate tumour entity and the most common sarcoma of the gastrointestinal tract. The term GIST was first used in 1983 by Mazur and Clark to encompass gastrointestinal non-epithelial neoplasms that lacked the immunohistochemical features of Schwann cells and did not have the ultrastructural characteristics of smooth muscle cells (1). GISTs continued to be rarely diagnosed until about the year 2000, when they became a focus of intense research. Hirota et al. (2) reported in 1998 that gain-of-function mutations in the KIT (c-kit) proto-oncogene are present in most GISTs. KIT gene encodes the KIT protein, which is the transmembrane receptor for the cytokine known as stem cell factor (SCF). The intracytoplasmic portion of KIT functions as a tyrosine kinase. GISTs were found to be generally resistant to cancer chemotherapy and associated with poor outcome, until in 2001 imatinib mesylate was found to be highly active against chemotherapy-resistant GIST (3). At present GISTs may be defined as morphologically spindle cell, epitheloid, or occasionally pleomorphic, mesenchymaltumours that usually arise from the gastrointestinal tract, usually express the KIT protein $(95 \%$ stain positively for KIT in immunohistochemistry) and that often harbour mutation of a gene that encodes for a type III receptor tyrosine kinase (either KIT or PDGFRA, up to $90 \%$ of the cases). The KIT protein is readily detectable by immunohistochemical assays, and the gene mutations can be detected by mutation analysis usually based on DNA sequencing. Other useful diagnostic features for GIST are negative immunostaining for desmin (>95\%), and absence of lymph node and lung metastases.

\section{Address for correspondence:}

V. Bozhkov, Second Dept. of Surgery, UMHAT "Sveta Marina" Varna, 1, Christo Smirnenski blvd., Varna 9010, Bulgaria.

e-mail: mrboj@abv.bg

\section{EPIDEMIOLOGY}

Epidemiology of GIST is incompletely known. Three studies that used up-to-date diagnostic criteria found the annual incidence of GIST to be 14.5 per million in south-west Sweden, 11 per million in Iceland, and 12.7 per million in the Netherlands (5-7). Approximately $10 \%$ of cases were detected at autopsy in these series, and $20 \%$ at endoscopy, imaging of the abdomen, or at surgery for other conditions. GISTs vary in malignancy potential ranging from small, incidentally detected tumours with excellent outcome to aggressive sarcomas. The proportion of overtly malignant or high-risk GISTs is $20-35 \%$ of all GISTs $(5,8)$ suggesting that the annual incidence of GISTs with a high malignancy potential is about 5 per million. It is likely that many small GISTs are not reported to cancer registries. A study based on the Surveillance, Epidemiology and End Results (SEER) registry data from the USA from 1992 to 2000 found the age-adjusted yearly incidence of GIST to be 6.8 per million (9).

GISTs might constitute approximately one sixth to one third of all soft tissue sarcomas, depending on whether there is an accounting for small and incidental GISTs. Many small, asymptomatic GISTs may remain undetected. In one study, two GISTs were found per 1000 autopsies performed (5), suggesting that asymptomatic GISTs of low malignancy potential may not be uncommon tumours in the elderly general population, and that these GISTs might be much more frequent than GISTs with a high malignancy potential. The frequency of reported GISTs may change with time because of evolving diagnostic criteria and the greater awareness of GIST (7).

\section{HISTOPATHOLOGY}

GISTs range in size from a few millimetres to $35 \mathrm{~cm}$, with a median size of between $5 \mathrm{~cm}$ and $8 \mathrm{~cm}(5,6)$. GISTs probably originate from the interstitial cells of Cajal or their pre- 
cursors (12). Microscopically, GIST cell morphology is usually spindle-shaped (70\%), but some GISTs consist of rounded cells (epithelioid type, 20\%) or a mixture, but they can also be pleomorphic (13). Large GISTs often show cystic degeneration or central necrosis.

Many GISTs have an uncertain malignancy potential. Estimation of the risk of recurrence will become important provided that adjuvant therapy becomes a standard praxis in the management. The most commonly used scheme to assess the risk of recurrence is the consensus approach, which is based on the primary tumour diameter and the mitotic count (Table. 1).

\section{HPF, high power field}

Patients whose tumour has ruptured into the abdominal cavity either before surgery or at surgery have a very high risk of tumour recurrence. On the other hand small $(<1 \mathrm{~cm})$, incidentally found GISTs behave, almost invariably, in a benign fashion. Tumours arising from the small bowel, colon, rectum or mesentery are generally associated with less favourable outcome than those arising from the stomach.

Table 1

\begin{tabular}{||l|c|c||}
\hline Risk category & Size & Mitotic count \\
\hline Very low risk & $<2$ ñì & $<5 / 50 \mathrm{HPF}$ \\
\hline Low risk & $2-5$ ñì & $<5 / 50 \mathrm{HPF}$ \\
\hline Intermediate risk & $<5$ ñì & $6-10 / 50 \mathrm{HPF}$ \\
\hline High risk & $\begin{array}{c}>10 \text { ñì } \\
\text { any size } \\
>5 \text { ñì }\end{array}$ & $\begin{array}{c}\text { Any mitotic rate } \\
>10 / 50 \mathrm{HPF} \\
>5 / 50 \mathrm{HPF}\end{array}$ \\
\hline
\end{tabular}

\section{Presenting symptoms and signs}

The median age at diagnosis is $66-69$ years in population-based studies. These series include tumours detected incidentally or at autopsy, which are generally found at an older median age $(5,6)$. In the SEER registry data the median age at the time of diagnosis was 63 years (9). Only 3\% of GISTs are diagnosed before the age of 21 years (10), and GISTs arise only exceptionally in children. In the SEER series $55 \%$ of GISTs occurred in males (9) whereas an equal gender distribution was found in a population-based analysis from Sweden as well as in a large series from Korea $(5,11)$. No predisposing factors have been described. The most common symptom at presentation is bleeding (10). Large GISTs often protrude from the site of origin and grow between the bowel loops and the abdominal organs, but they may also erode the gastrointestinal tract lumen. Bleeding may take place either into the abdominal cavity causing acute abdominal pain and severe anaemia and sometimes leading to emergency surgery, or into the gastrointestinal tract lumen causing haematemesis, melena and anaemia. Patients with GIST may also have various other symptoms, such as abdominal pain or discomfort, early satiety, bloating, obstructive jaundice, dysphagia, fever and anaemia-related symptoms such as fatigue and palpitations (10), or they may present with an abdominal tumour with no symptoms. Between $10 \%$ and $25 \%$ of patients present with metastatic disease $(5,9)$. GISTs can originate anywhere in the GI tract. The stomach (40-60\%) and small intestine $(30-40 \%)$ are the most common locations. The colon, rectum, oesophagus, and rarely mesentery, retroperitoneum and other intra-abdominal organs are other sites of origin. In the SEER data, $51 \%$ of the cases arose from the stomach, $36 \%$ from the small intestine, $7 \%$ from the colon, $5 \%$ from the rectum, and $1 \%$ from the oesophagus (9). Based on a multi-institutional survey from Korea, differences in clinical and histological features of GIST between Korean and Western populations appear to be minor (11). GISTs frequently give rise to numerous intra-abdominal metastases located on the peritoneal, omental, mesenteric and other serosal surfaces, and to liver metastases, whereas extraabdominal metastases are rare. In the late phases of advanced disease this results in a characteristic clinical presentation with a greatly enlarged abdomen coupled with muscle wasting. GISTs have a high tendency to seed. The intra-abdominal lesions probably result from tumour cell seeding into the abdominal cavity, whereas liver metastases probably from haematogenous spread. GIST patients may have metastases in surgical scars and sometimes even in needle tracts. Lymph node metastases are rare.

\section{DIAGNOSIS}

As part of the analysis, blood tests and CT scanning are often undertaken (Fig.1, Fig. 2.).

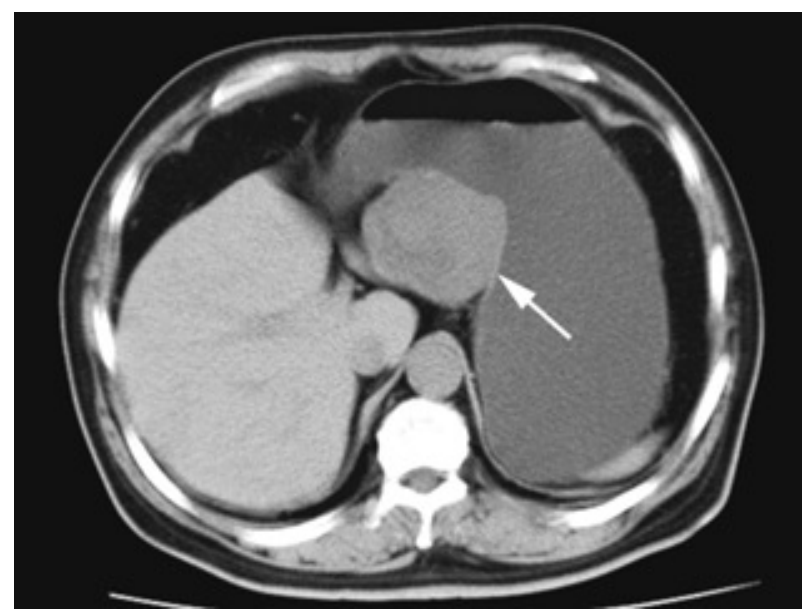

Fig. 1. Transverse CT scan image of upper abdomen showing an exophytic homogeneous mass (arrow).

\section{TREATMENT}

Surgery is the standard treatment for non-metastatic GISTs. The tumour should be removed en-bloc with its pseudocapsule to yield an adequate resection margin. The optimal width of the tumour-free margin has not been de- 
fined. Regional lymph node resection is of unproven value, since GISTs rarely give rise to lymph node metastases. Extensive lymphadenectomy is not recommended.

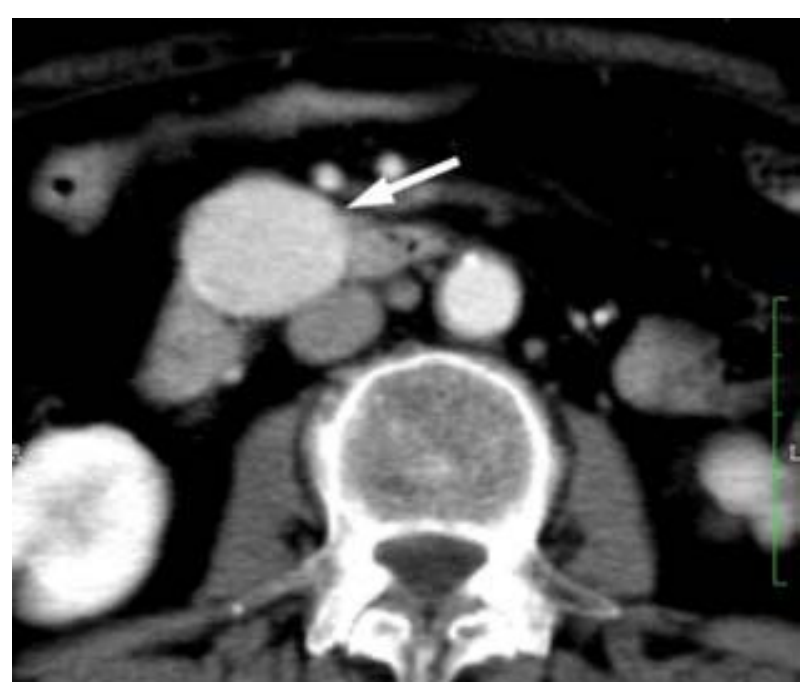

Fig. 2. An enhanced CT scan of the upper abdomen shows an enhancing extrophytic heterogeneous mass (arrow), with central necrosis and a fluid level (long arrow), and leakage of the contrast agent (arrowhead).

Tumor rupture is associated with an increased risk for development of peritoneal implants. En-bloc resection is recommended whenever feasible in cases where contiguous organs are involved. A diagnostic coarse needle biopsy performed through the abdominal wall may increase the risk of tumour cell seeding into the abdominal cavity, which can often be avoided when a needle biopsy is taken at endoscopy.

It is highly recommended for patients who underwent for a GIST to have a healthy way of living, including normal weight, lack of bad habits and moderate physical activity. The standard of care after complete resection of a primary tumor is observation. During the first two years after surgical resection, a reasonable follow up would be a CT scan or ultrasound sonography every 3 months, then every 6 months next two years, and yearly thereafter.

Gastrointestinal stromal tumors are rare neoplasm thought to arise from mesenchymal cell of the gastrointestinal tract. The histogenesis, classification, diagnostic criteria and biologic behavior of GISTs have been the subject of much controversy. Recent studies include a variety of specialists surgeons, pathologists, molecular biologists and genetics. The early diagnosis is one of the most important features in correct therapeutical behavior of the GISTs. It is important to note that surgery remains the golden standard in treatment of GISTs, followed by an adjuvant therapy course and the prognosis is relevant with the stage of the disease.

The incidence of GIST is unknown, based upon the literature it is the same in all geographical and ethnic groups, in males and females and it appears mostly in $5^{\text {th }}-7^{\text {th }}$ decade of life. GIST represents $1 \%$ of all primary tumors of gastroin- testinal tract; it means they are detected rarely - most by an autopsy. In this aspect we should emphasize on early diagnosis and correct treatment.

\section{REFERENCES}

1. Mazur MT, Clark HB. Gastric stromal tumors: Reappraisal of histogenesis. Am J Surg Pathol 1983; 7: 507-519.

2. Hirota S, Isozaki K, Moriyama $\mathrm{Y}$ et al. Gain-of-function mutations of c-kit in human gastrointestinal stromal tumors. Science 1998; 279: 577-580.

3. Joensuu H, Roberts P, Sarlomo-Rikala M et a1. Effect of the tyrosine kinase inhibitor STI571 in a patient with metastatic gastrointestinal stromal tumor. N Engl J Med 2001; 344: 1052-1056.

4. Heinrich MC, Corless, CL, Duensing A et al. PDGFRA activating mutations in gastrointestinal stromal tumors. Science 2003; 299: 708-710.

5. Nilsson BP, Bumming P, Meis-Kindblom JM et al. Gastrointestinal stromal tumors: The incidence, prevalence, clinical course, and prognostication in the preimatinib mesylate era. Cancer 2005; 103: 821-829.

6. Tryggvason $\mathrm{G}$, Gislason HG, Magnusson MK et al. Gastrointestinal stromal tumors in Iceland, 1990-2003: The Icelandic GIST study, a population-based incidence and pathologic risk stratification study. Int J Cancer 2005; 117:289-293.

7. Goettsch WG, Bos SD, Breekveldt-Postma N et al. Incidence of gastrointestinal stromal tumours is underestimated: Results of a nation-wide study. Eur $J$ Cancer 2005; 41: 2868-2872.

8. Miettinen M, Lasota J. Gastrointestinal stromal tumors-definition, clinical, histological, immunohistochemical, and molecular genetic features and differential diagnosis. Virchows Archiv 2001; 438: $1-12$.

9. Tran T, Davila JA, El-Serag HB. The epidemiology of malignant gastrointestinal stromal tumors: an analysis of 1458 cases from 1992 to $2000 . A m J$ Gastroenterol 2005; 100: 162-168.

10. Miettinen M, Sobin LH, Lasota J. Gastrointestinal stromal tumors of the stomach: a clinicopathologic, immunohistochemical, and molecular genetic study of 1765 cases with long-term follow-up. Am J Surg Pathol 2005; 29: 52-68.

11. Kim KM, Kang DW, Moon WS et al. Gastrointestinal Stromal Tumors in Koreans: Incidence and the Clinical, Pathologic and Immunohistochemical Findings. J Korean Med Sci 2005; 20: 977-984.

12. Kindblom LG, Remotti HE, Aldenborg F et al. Gastrointestinal pacemaker cell tumor (GIPACT): gastrointestinal stromal tumors show phenotypic characteristics of the interstitial cells of Cajal. Am J Pathol 1998; 152: 1259-1269.

13. Fletcher CD, Berman JJ, Corless C et al. Diagnosis of gastrointestinal stromal tumors: A consensus approach. Hum Pathol 20. 